\title{
Digital Radio Mondiale (DRM) Measurement System Design and Measurement Methodology for Fixed and Mobile Reception
}

\author{
G. Prieto, D. Guerra, J. M. Matías, M. M. Vélez and A. Arrinda \\ University of the Basque Country - UPV/EHU \\ Alda. Urquijo S/N, 48013 BILBAO, Spain \\ \{gorka.prieto, david.guerra, josemaria.matias, manuel.velez, amaia.arrinda\}@ehu.es
}

\begin{abstract}
This paper presents a measurement system design and the measurement techniques developed to study DRM (Digital Radio Mondiale) signal behavior and system performance. The solution proposed considers different reception conditions and environments. Some aspects, like antenna selection and both internal and external noise measurement, have turned to be key factors and are also presented.
\end{abstract}

Index Terms-Measurement system, mobile measurements, external noise, receiver sensitivity, DRM.

\section{INTRODUCTION}

D RM (Digital Radio Mondiale) is the standard adopted in Europe for digital audio broadcasting in the frequency bands below $30 \mathrm{MHz}$ [1]-[3]. The work of developing all the technical, promotional and standardization tasks has been mainly carried out by the members of the DRM consortium [4]. The result of this collaborative effort is a successful launch of commercial emissions in June 2003 by the 16 leading international broadcasters.

Despite this successful launch of services, there is still a need for network parameter planning values measured on the field [5], [6], and also to analyze the behavior of the DRM system under several reception environments and conditions. In order to perform this measurement campaign as efficiently and productively as possible, both a measurement mobile unit and a special measurement methodology have been designed.

DRM allows a number of parameters to be adjusted to provide a better performance for the different propagation cases and reception conditions that can occur using these frequency bands [7], [8]. These parameters range from OFDM related parameters, such as number of carriers and guard interval, to symbol and bit-stream related parameters, such as interleaver depth and protection ratios. The designed measurement methodology takes this into account. It allows to remotely change the transmitter parameters from the mobile unit in order to test different signal configurations and also to perform different kinds of measurements.

Some important conclusions were also obtained while implementing the measurement system and performing the first tests. Man made noise has a great impact on reception quality and is a key factor when choosing a suitable antenna for DRM

UPV/EHU is a member of the System Evaluation (TC-SE) and Monitoring Network (TC-MN) groups of the DRM Consortium. reception. Noise measurement has turned to be a need, and suitable equipment configuration and measurement methodology has been designed.

\section{Measurement System}

\section{A. Measurement Targets}

The measurement system presented in this document has been designed with the aim of studying a range as wide as possible of aspects for this emerging technology, and the low bandwidth of the DRM signal has contributed to this goal.

Among these aspects, the targeted ones are the following:

- A first aspect to study is the evaluation of suitable channel propagation models. This evaluation implies obtaining the time and spatial behavior of the received signal level.

- A second aspect is the characterization of fixed reception parameters. This characterization includes a minimum SNR and minimum field strength requirement measurement and its comparison to the initial values obtained from laboratory simulations and recommended by the ITU-R [9].

- Another key aspect to study is the characterization for mobile reception. Relevant measurements in this mobile reception are the effects of power lines and tunnels depending on the vehicle speed.

- All these measurements must be done taking into account different reception environments such as rural, residential and commercial.

- Finally the system must be able to dynamically modify the parameters of the transmitted signal to measure their effect on the reception experience.

\section{B. Measurement System Design}

The DRM measurement system that has been designed is depicted in figure 1. The measurement system allows to be installed on a van in order to carry out a measurement campaign with a mobile unit. From figure 1 it can be seen that the system is composed of three different sections: the acquisition and distribution section, the measurement section and the control section.

The acquisition and distribution section is composed of the Rohde\&Schwarz HE010 short monopole active antenna 


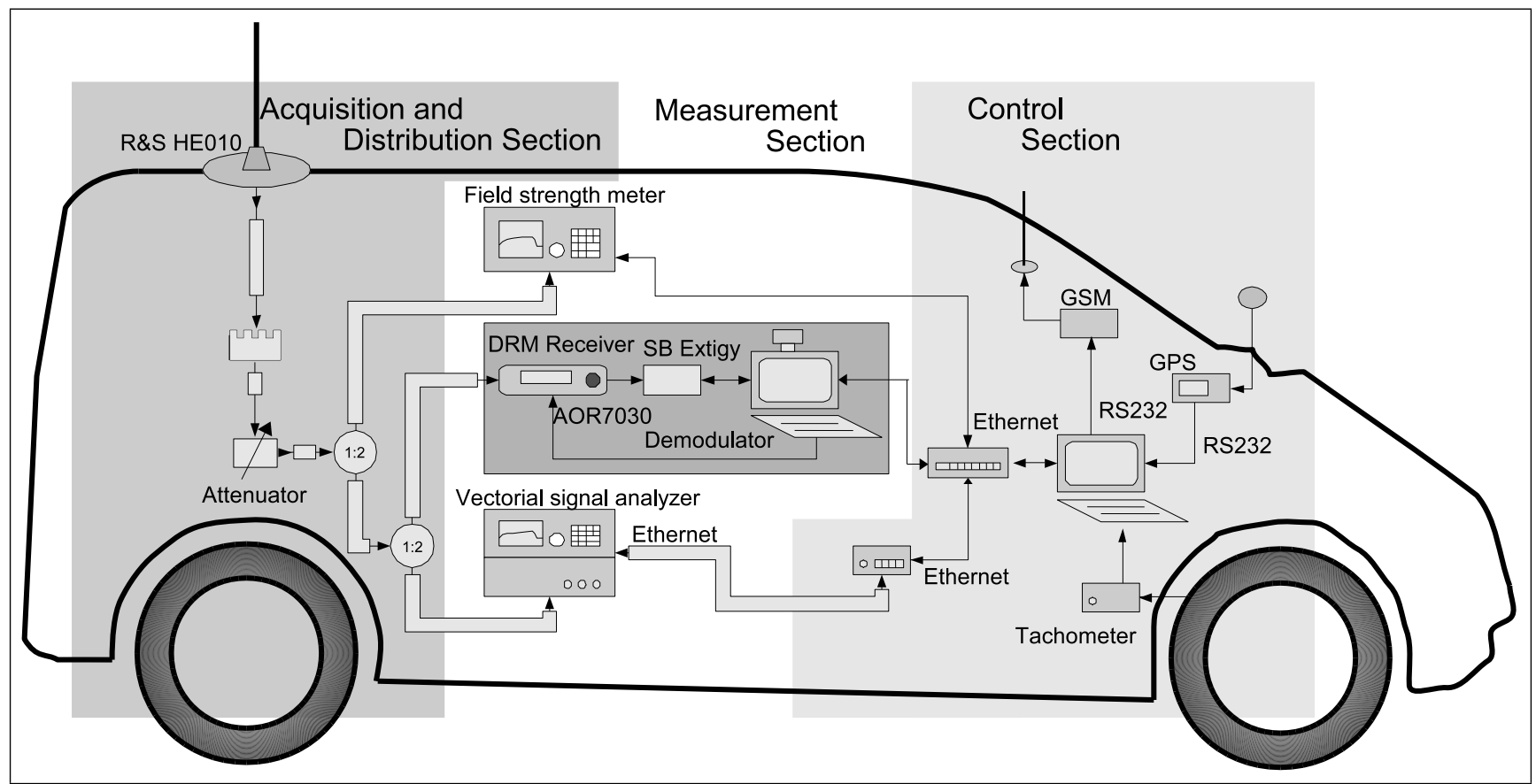

Fig. 1. DRM Measurement System Architecture

with a $40 \mathrm{MHz}$ low-pass filter modification in order to avoid undesired out of band interferences. The antenna is placed at the top of the van with a specifically prepared ground plane. Then the received signal passes though a variable logarithmic attenuator, and the distribution to the rest of the equipment is done using two power splitters.

The measurement section is composed of two main blocks. The first one (the DRM receiving chain) is composed of the AOR7030 analogue front-end, capable of tuning the received signal and down-convert it to a $12 \mathrm{kHz}$ intermediate frequency. The next component in the chain is a SB Extigy external sound card responsible of sampling the $12 \mathrm{kHz}$ IF signal and sending the samples to a PC, which is the actual DRM demodulator. For this purpose the professional version of the FhG software radio has been used.

The other block of the measurement section is responsible of measuring the field strength and capturing the RF spectra. It is composed of a vectorial signal analyzer to provide spectrum traces and a field strength meter to measure the RF signal power.

The last section is the control system, responsible of configuration and control of all the equipment, data storage, online statistics calculation and position measurement. This section also provides a GUI to a human user. The control system is based on a laptop running a designed software on a GNU/Linux platform.

This section includes auxiliary devices such as a GPS and a tachometer trigger system, which provides a precise reference to calculate wavelength based statistics for mobile measurements. The tachometer also provides a backup of the GPS in case this last one fails, as happens inside tunnels and places of bad coverage.

In order to allow to change the transmitted signal parameters from the mobile unit, a remote control system has been implemented. It is based on a call server located in the transmitter, ready to receive and answer GSM data calls from the measurement mobile unit. This process is automated via UNIX shells scripts which connect the modem to the network and execute remote commands via SSH. The access to the GSM network is done via two identical modules located in the van and in the transmitter.

\section{Antenna Selection}

Antenna selection has turned to be a key factor in order to achieve good measurement results for both signal and noise, specially for urban environments.

Two types of antennas have been considered. A passive magnetic transfer helical antenna and a R\&S HE010 short monopole active antenna. The active one has two main advantages: it is characterized by the manufacturer, and has a wider frequency response. But this wide frequency response has a big counterpart since FM transmissions up to $100 \mathrm{MHz}$ are also amplified. This leads to antenna saturation noise in environments with high signal power out of the DRM band, such as FM transmissions in urban environments. For these later cases the passive antenna solves the problem, but this antenna is not characterized. The solution adopted consists on using the active antenna but with a $40 \mathrm{MHz}$ limiter before the feeder, thus filtering non-DRM signals.

Figure 2 shows an AM spectrum measured with the mentioned antennas in a noisy urban environment. From this figure, the antenna saturation noise effect is clear for the original active antenna. The antenna with the $40 \mathrm{MHz}$ limiter, on the contrary, does not suffer from this saturation noise and amplifies both signal and noise above the internal measurement equipment noise. The passive antenna logically also avoids 


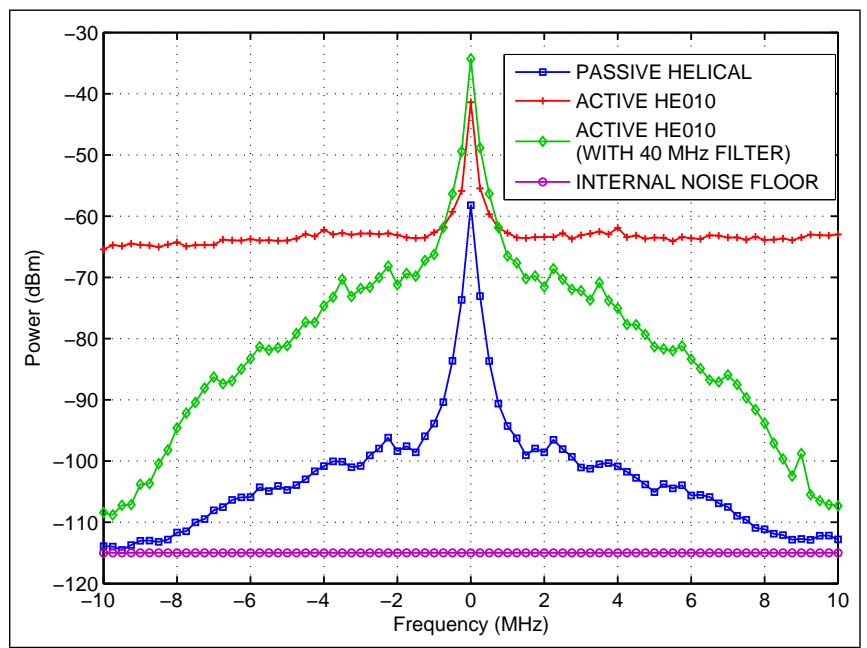

Fig. 2. Measured AM spectrum in a noisy urban environment

saturation noise, but gives signal values closer to the measurement equipment internal noise floor.

\section{Internal Noise Floor}

It is very important to characterize the internal noise floor of the measurement system in order to determine the smallest signal level than can be measured with it. If the received signal level is close to the internal noise floor of the equipment, the measured level would be distorted.

The two key elements of the presented DRM measurement system that have to be analyzed are the field strength meter and the DRM receiver. The procedure that has been used for measuring the internal noise floor for both equipments consists on generating a DRM signal to feed the system and vary its power in $1 \mathrm{~dB}$ steps.

The field strength meter is expected to initially provide measured values in steps of $1 \mathrm{~dB}$, but these steps would become smaller as the input signal level approaches to the internal noise floor.

In relation to the DRM receiver, which consists on the analogue front-end and the sound card, this absolute signal level value cannot be measured. In this case, the signal to noise ratio of the samples captured by the sound card is used instead. This ratio should remain constant until the external noise level reaches the internal noise floor of the receiver, starting then to decrease until being zero.

This behavior has been measured and its depicted in figure 3 for both the field strength meter and the DRM receiver.

Since both the DRM signal and the noise signal have the same distribution (gaussian) and are uncorrelated, the power level measured by the field strength meter for the resulting signal is the lineal sum of the power of each of the signals. Equation (1) shows this sum in logarithmic scale.

$$
\begin{gathered}
P_{M}=10 \cdot \log \left(10^{\frac{P_{R}}{10}}+10^{\frac{N}{10}}\right) \\
\text { where } \begin{cases}P_{M} & \text { measured power } \\
P_{R} & \text { real power } \\
N & \text { internal noise floor }\end{cases}
\end{gathered}
$$

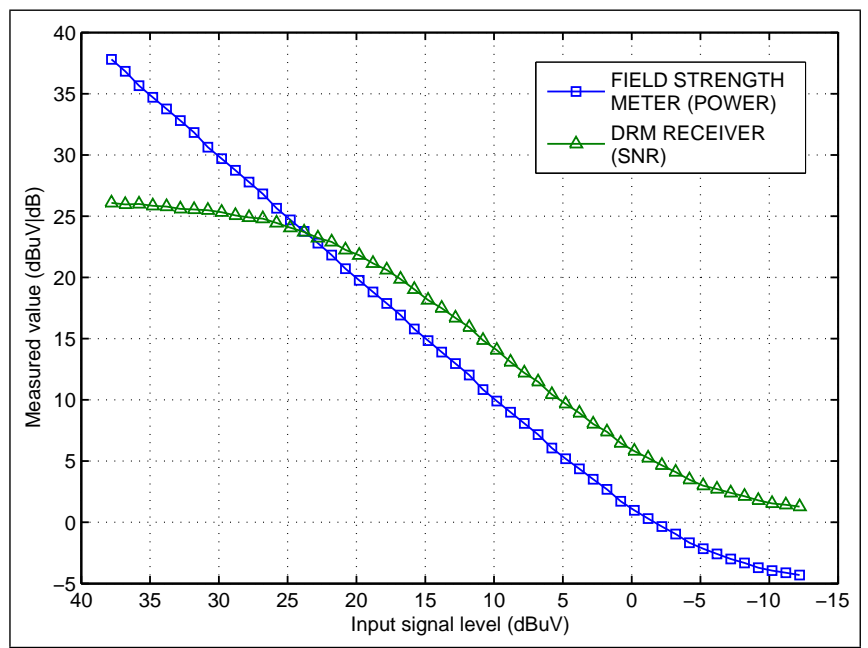

Fig. 3. Effect of internal noise floor

For the case of the DRM receiver, the measured SNR value is affected by both the internal noise floor and the external noise level. Applying a similar reasoning to the one presented for the field strength meter, equation (2) is obtained for describing the behavior of the measured SNR value in logarithmic scale.

$$
\begin{gathered}
S N R=10 \cdot \log \left(10^{\frac{S}{10}}+10^{\frac{R}{10}}+10^{\frac{N}{10}}\right) \\
-10 \cdot \log \left(10^{\frac{R}{10}}+10^{\frac{N}{10}}\right) \\
\text { where } \begin{cases}S N R & \text { measured signal to noise ratio } \\
S & \text { signal power } \\
N & \text { internal noise floor } \\
R & \text { external noise power }\end{cases}
\end{gathered}
$$

The method designed for working out the internal noise floor values for both the field strength meter and the DRM receiver consist on applying a MMSE criterion. The obtained noise values minimice the MSE between the theoretical behavior described by (1) and (2) and the measured values depicted in figure 3.

The results of applying this procedure are depicted in figures 4 and 5 for the field strength meter and the DRM receiver respectively. The obtained values are summarized in table I. These values are referenced to the input of the measurement system, to reference it to the input of their corresponding equipments, the attenuation inserted by the distribution system must be subtracted. This attenuation is $6 \mathrm{~dB}$ for the DRM receiver and $12 \mathrm{~dB}$ for the field strength meter.

\begin{tabular}{l||c|c}
\hline & $\begin{array}{c}\text { Field strength } \\
\text { meter }\end{array}$ & $\begin{array}{c}\text { DRM } \\
\text { receiver }\end{array}$ \\
\hline \hline $\begin{array}{l}\text { Internal } \\
\text { noise floor }\end{array}$ & $-5.1 \mathrm{~dB} \mu \mathrm{V}$ & $-4.1 \mathrm{~dB} \mu \mathrm{V}$ \\
\hline $\begin{array}{l}\text { Noise } \\
\text { figure }\end{array}$ & $22.3 \mathrm{~dB}$ & $23.3 \mathrm{~dB}$ \\
\hline \multicolumn{3}{c}{ TABLE I }
\end{tabular}

MEASURED NOISE FLOOR AND NOISE FIGURES 


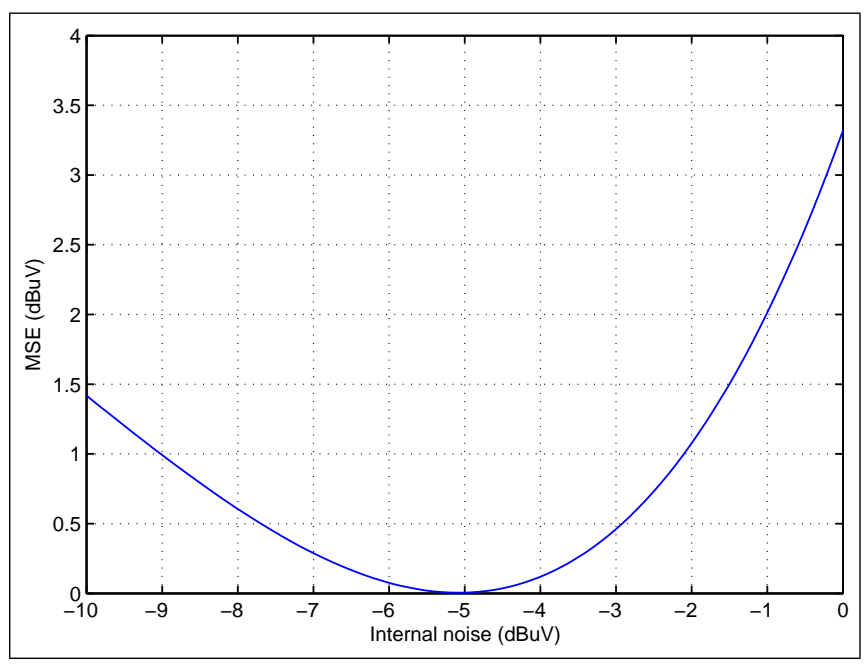

Fig. 4. MMSE results for the field strength meter

Finally it must be pointed out that, in order to achieve these small noise values for the case of the field strength meter, it has been necessary to switch on its internal preamplifier of $20 \mathrm{~dB}$ and switch off its input attenuator of $10 \mathrm{~dB}$.

\section{Measurement Methodology}

\section{A. Measurement Parameters}

Part of the desired measurement parameters are provided by the DRM receiver via a standardized interface but, beside these parameters, additional measurements must be done for RF analysis and also for logging position and speed information.

The measurement parameters that a DRM professional receiver should provide are standardized by the RSCI (Receiver Status and Control Interface) specification [10]. These parameters can be accessed via DCP (Distribution and Communications Protocol) which is also standardized [11].

RSCI provides a quite wide range of parameters which include: channel measurements like delay and Doppler spread, constellation measurements like MER, and baseband measurements like number of errored bits and audio dropout patterns. All of these parameters are provided every $400 \mathrm{~ms}$, which is the duration of a DRM frame, and are recorded by the measurement system.

Due to this time constraint, this $400 \mathrm{~ms}$ interval has been chosen as the base measurement interval for the rest of the equipment in the system. In addition to all the RSCI measurements, the DRM signal strength is also measured integrating the received level during $400 \mathrm{~ms}$ (longer than a symbol duration) using a RMS detector. For the case of fixed measurements a measurement duration of several minutes is recommended to take into account the influence of external agents like traffic passing near the measurement unit.

For the case of mobile measurements a tachometer trigger has been designed in order to provide a precise reference to calculate wavelength based measurements. These measurements are recorded every a preconfigured number of turns of the van wheel, which defaults to a sampling distance of $\lambda / 10$. This spacing is considered small enough to study which could be a recommended spacing value in order to avoid

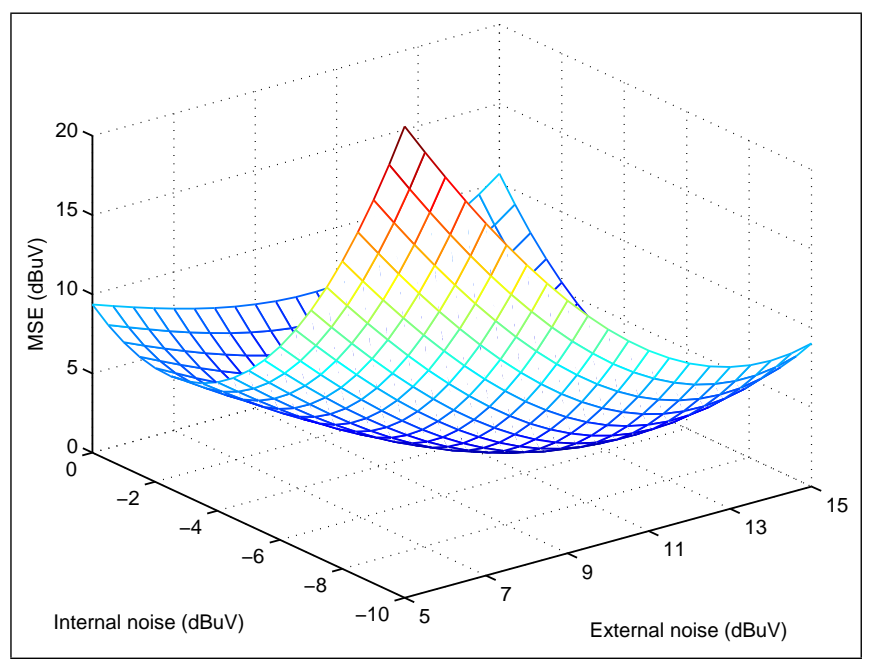

Fig. 5. MMSE results for the DRM receiver

waveform sampling redundancy at these low frequencies. Studies performned for other frequency ranges have obtained recommended sampling distances of $0.9 \lambda$ [12] and $0.38 \lambda$ [13].

\section{B. SNR Measurement}

One parameter of special interest for a broadcasting networks planning tool is the minimum requirement in the ratio between the modulated signal RF power and the external noise level. This is what is denoted in this section as minimum SNR requirement.

A common procedure for measuring the minimum SNR requirement consists on attenuating the input signal of the system until a threshold BER (Bit Error Rate) is measured on the receiver. But this is not suitable for all the cases under study. When the DRM signal uses ground wave propagation (typical of the medium wave band during daytime), there are not multipath or Doppler effects that decrease signal quality, instead the channel behaves as a Gaussian one. Thus the main cause of decrease on signal quality in this case is a low signal level or a high external noise level.

From this consideration it is concluded that attenuating the system input would attenuate both input signal and external input noise resulting in the same SNR measurement. This would last until the attenuated input noise is lower than the measurement equipment internal noise floor, in which moment the measured SNR begins to decrease. This is shown in figure 6.

This figure shows how SNR, and thus BER, remains constant until the equipment internal noise is reached. This is not the desired measurement since the minimum SNR requirement would be measured in relation to the internal noise floor of the receiver, instead of in relation to the external noise level of the reception environment.

From this consideration it is concluded that finding locations with the desired SNR or BER range would be a much better solution than attenuating the input signal. This would result on a set of BER versus SNR measurements corresponding to different locations, and then an interpolation algorithm as the 


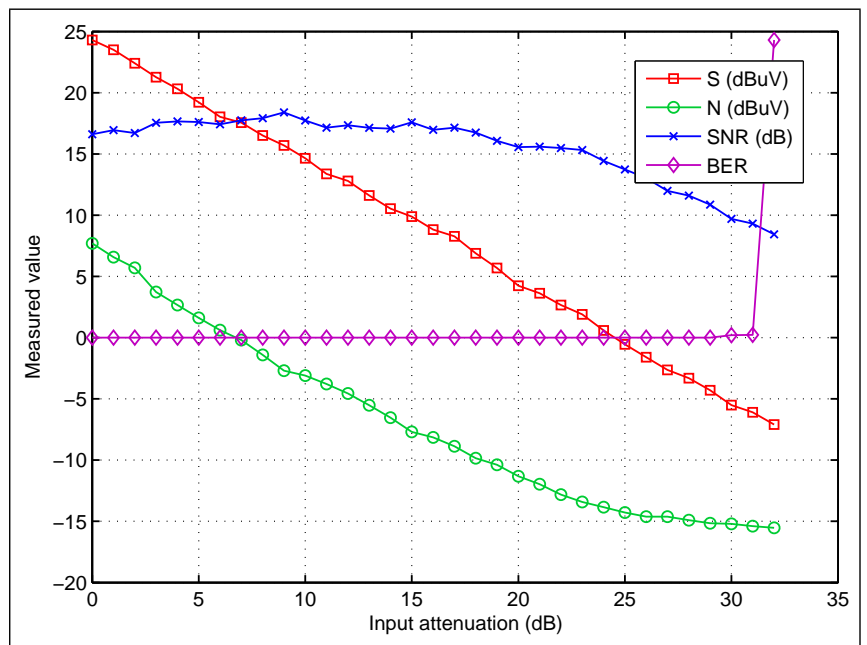

Fig. 6. Attenuating system input attenuates both signal and external noise

one described in [14] could be used to obtain a minimum SNR requirement for a given threshold BER.

\section{External Noise Measurement}

This measurement system has been designed in such a way that can be used to make external noise measurements in the DRM bands.

As explained in section II-C the $40 \mathrm{MHz}$ limiter before the antenna feeder avoids interference of strong signals out of the DRM bands. Furthermore, the antenna generated noise [15] is supposed to be $10 \mathrm{~dB}$ below the expected value for external noise levels [7].

In relation to the internal noise floor of the measurement system, the results presented in section II-D have been obtained for the medium wave band. These values are small enough in comparison to external noise levels of the medium wave band. For the case of other DRM bands, a study similar to the one presented should be performed to assure good measurement results.

\section{CONCLUSION}

This paper has presented a measurement system design and a measurement methodology optimized for testing and characterization of the DRM signal behavior and performance. Since this is an emerging technology, carrying out measurement campaigns is something undoubtedly necessary. These measurements allow to test the system performance and provide a guidance for planning values to be recommended.

The system presented in this paper has been of a great help to achieve these tasks and has been successfully used in the first two DRM measurement campaigns for the medium wave band carried out in Spain [16], [17].

It is also important to point out that the antenna and noise considerations presented in this paper are of crucial importance in order to achieve quality measurement results.

\section{ACKNOWLEDGMENT}

The authors would like to thank to the colleagues within the DRM consortium for their suggestions and help.

This work has been economically supported by public funding under the scope of the projects MCYT TIC2002-01340 and UPV/EHU-UE2003.

\section{REFERENCES}

[1] System for digital sound broadcasting in the broadcasting bands below $30 \mathrm{MHz}$, International Telecommunications Union ITU-R Recommendation BS.1514-1, Oct. 2002.

[2] Digital Radio Mondiale (DRM) - Part 1: System specification, International Electrotechnical Commission Std. IEC 62 272-1, Mar. 2003.

[3] Digital Radio Mondiale (DRM); System Specification, European Telecommunications Standards Institute Std. ETSI ES 201980 v2.2.1, Oct. 2005.

[4] Official DRM website. [Online]. Available: http://www.drm.org

[5] F. Hofmann, C. Hansen, and W. Schafer, "Digital radio mondiale (DRM) digital sound broadcasting in the AM bands," IEEE Trans. Broadcast., vol. 49, no. 3, pp. 319-328, Sept. 2003.

[6] J. Briggs, "DRM - a summary of the field trials," European Broadcasting Union (EBU), Technical Review trev-296, Oct. 2003.

[7] Radio noise, International Telecommunications Union ITU-R Recommendation P.372-8, Apr. 2003.

[8] Propagation factors affecting systems using digital modulation techniques at $L F$ and $M F$, International Telecommunications Union ITU-R Recommendation P.1321, Aug. 1997.

[9] Planning parameters for digital sound broadcasting for frequencies below $30 \mathrm{MHz}$, International Telecommunications Union ITU-R Recommendation BS.1615, 2003.

[10] Digital Radio Mondiale (DRM); Receiver Status and Control Interface (RSCI), European Telecommunications Standards Institute Std. ETSI TS 102349 v1.1.1, Jan. 2005.

[11] Digital Radio Mondiale (DRM); Distribution and Communications Protocol (DCP), European Telecommunications Standards Institute Std. ETSI TS 102821 v1.1.1, Dec. 2003.

[12] W. C. Y. Lee, "Estimate of local average power of a mobile radio signal," IEEE Trans. Veh. Technol., vol. 34, no. 1, Feb. 1985.

[13] D. Parsons, The Mobile Radio Propagation Channel. John Wiley \& Sons, 1992, ISBN 0-471-96415-8.

[14] G. Prieto, M. M. Vélez, P. Angueira, D. Guerra, and D. de la Vega, "Minimum C/N requirements for DRM reception based on field trials," IEEE Commun. Lett., vol. 9, no. 10, pp. 877-879, Oct. 2005.

[15] Manual Active Rod Antenna HE010, Rohde\&Schwarz, 1997, Order No. 0523.1414.

[16] D. Guerra, G. Prieto, I. Fernández, J. M. Matías, and P. Angueira, "Medium wave DRM field test results in urban and rural environments," IEEE Trans. Broadcast., vol. 51, no. 4, pp. 431-438, Dec. 2005.

[17] G. Prieto, M. M. Vélez, P. Angueira, D. Guerra, D. de la Vega, and A. Arrinda, "Digital Radio Mondiale (DRM). Field Trials for Minimum C/N Requirements," in Proc. of the International Broadcasting Convention, IBC 2005, vol. 1, Amsterdam, The Netherlands, Sept. 8-13, 2005, pp. 43-48.

All Authors are members of the TSR (Signal Processing and Radiocommunications) Research Group. This group belongs to the Department of Electronics and Telecommunications at the Bilbao Engineering College (University of the Basque Country) and focuses its main interest on digital broadcasting systems. 